\title{
Investigation of the Evolution of Magnetic and Acoustic Characteristics as a Result of Annealing of Cold-Deformed Nickel
}

\author{
Vladimir Kostin ${ }^{1,}$, Evgeny Serbin ${ }^{1}$, and Olga Vasilenko $^{1}$ \\ ${ }^{1}$ M.N. Mikheev Institute of Metal Physics of Ural Branch of Russian Academy of Sciences, 620137 \\ Yekaterinburg, Russian Federation
}

\begin{abstract}
The change in the magnetic and acoustic properties of nickel as a result of cold deformation and subsequent annealing is investigated. It was found that annealing leads to very significant changes in the magnetic and acoustic properties of nickel. The coercive force decreases 50 times with an increase in the annealing temperature to $800^{\circ} \mathrm{C}$. The Rayleigh coefficient, which characterizes the mobility of domain walls, monotonically increases by a factor of 20 over the entire investigated range of annealing temperatures. The greatest changes in the velocity and damping of longitudinal ultrasonic vibrations occur in the range of recrystallization temperatures. It is shown that to diagnose the structure and magnitude of residual stresses in nickel, it is advisable to determine its magnetic and acoustic parameters.
\end{abstract}

\section{Introduction}

In works [1-6] and a number of other works it was shown that the determination of the set of magnetic and magnetoacoustic characteristics makes it possible to reliably assess the structural-phase state, strength properties, as well as the stresses present in ferromagnetic materials. This possibility is due to the following reasons: 1) the interaction of moving domain boundaries with defects in the crystal structure and inhomogeneous stresses; 2) magnetoelastic effect - a change in magnetization and a change in the dynamics of 90degree domain walls when elastic stresses are applied to a ferromagnet; 3) magnetostrictive effect - a local change in the size of the ferromagnet due to the rearrangement of the domain structure. The latter effect is the main cause of magnetoacoustic emission (MAE), which is understood as a set of elastic vibrations arising in a ferromagnet during its magnetization reversal.

Recently, the use of nickel-based coatings and alloys has been expanding, and diagnostic methods for the corresponding materials and objects are being developed.

Thus, the purpose of this work was to determine the relationship between the magnetic and acoustic characteristics of nickel subjected to cold plastic deformation and subsequent annealing in order to identify promising diagnostic parameters for assessing its stress-strain state.

\footnotetext{
*Corresponding author: kostin@imp.uran.ru
} 


\section{Samples and measurement procedures}

Samples for research were made of commercially pure nickel. The workpieces were deformed by $60 \%$ by pulling through the dies according to the change in the cross-sectional area. Then, by grinding, they were brought to dimensions of $5.38 \times 5.38 \times 65.0 \mathrm{~mm}$ and annealed at various temperatures for 1 hour.

The magnetic properties were measured using a REMAGRAPH C-500 hysteresis graph complex in a closed magnetic circuit, i.e. the properties of the substance were measured [7].

The measurement of the velocity of longitudinal ultrasonic vibrations in cold-deformed and annealed nickel was carried out with an UD9812 "Uralets" ultrasonic flaw detector with a direct piezoelectric transducer P111-5.0-K8 with a frequency of $5 \mathrm{MHz}$. Sounding was carried out from the end surface of the samples.

The ultrasound damping measurement technique consisted in measuring the amplitudes of four consecutive bottom signals and further calculating the damping coefficient $\delta$ according to the formula:

$$
\mathrm{A}=\mathrm{A}_{0} \mathrm{e}^{-\delta \mathrm{r}}
$$

where $\mathrm{A}$ and $\mathrm{A}_{0}$ - amplitudes of adjacent bottom signals, [V],

$\mathrm{r}$ - the path traveled by the wave (double thickness $\mathrm{h}$ of the sample), [m]

The damping coefficient $\delta$ was calculated using the formula:

$$
\delta=\left(\mathrm{A}-\mathrm{A}_{0}\right) / \mathrm{r} \text {, }
$$

where, $\mathrm{r}=2 \mathrm{~h}$

\section{Discussion of the results}

The dependences of the magnetic properties on the annealing temperature of cold-deformed nickel are shown in Fig. 1. It is seen that annealing has the greatest effect on the coercive force $\left(\mathrm{H}_{\mathrm{c}}\right)$ of nickel. With an increase in the annealing temperature to $800{ }^{\circ} \mathrm{C}$, the $\mathrm{H}_{\mathrm{c}}$ value decreases by more than 50 times. Such a significant change is due to the combined effect of a decrease in internal stresses, a decrease in the dislocation density, and an increase in the average grain size as a result of recrystallization. All these factors lead to an increase in the magnitude of magnetic domains in the material and an increase in the mobility of domain walls $[7,8]$. This is confirmed by the simultaneous growth of the maximum magnetic permeability ( $\mu$ max) and the Rayleigh coefficient $(\beta)$, which characterize the dynamics of magnetization of the material $[7,8]$. Residual magnetic induction $\left(\mathrm{B}_{\mathrm{r}}\right)$ decreases by about 1.5 times at annealing temperatures above $600{ }^{\circ} \mathrm{C}$, which corresponds to an increase in the size of recrystallized grains and facilitates the appearance and growth of nuclei of a reverse magnetic phase [7, 8]. An increase in $\mathrm{T}_{\text {ann }}$ from $800{ }^{\circ} \mathrm{C}$ to $900{ }^{\circ} \mathrm{C}$ leads to an almost twofold increase in the coercive force and a similar decrease in the maximum magnetic permeability. The most probable reason for such changes is an increase in macrostresses in the material due to sharp cooling from high temperatures. 

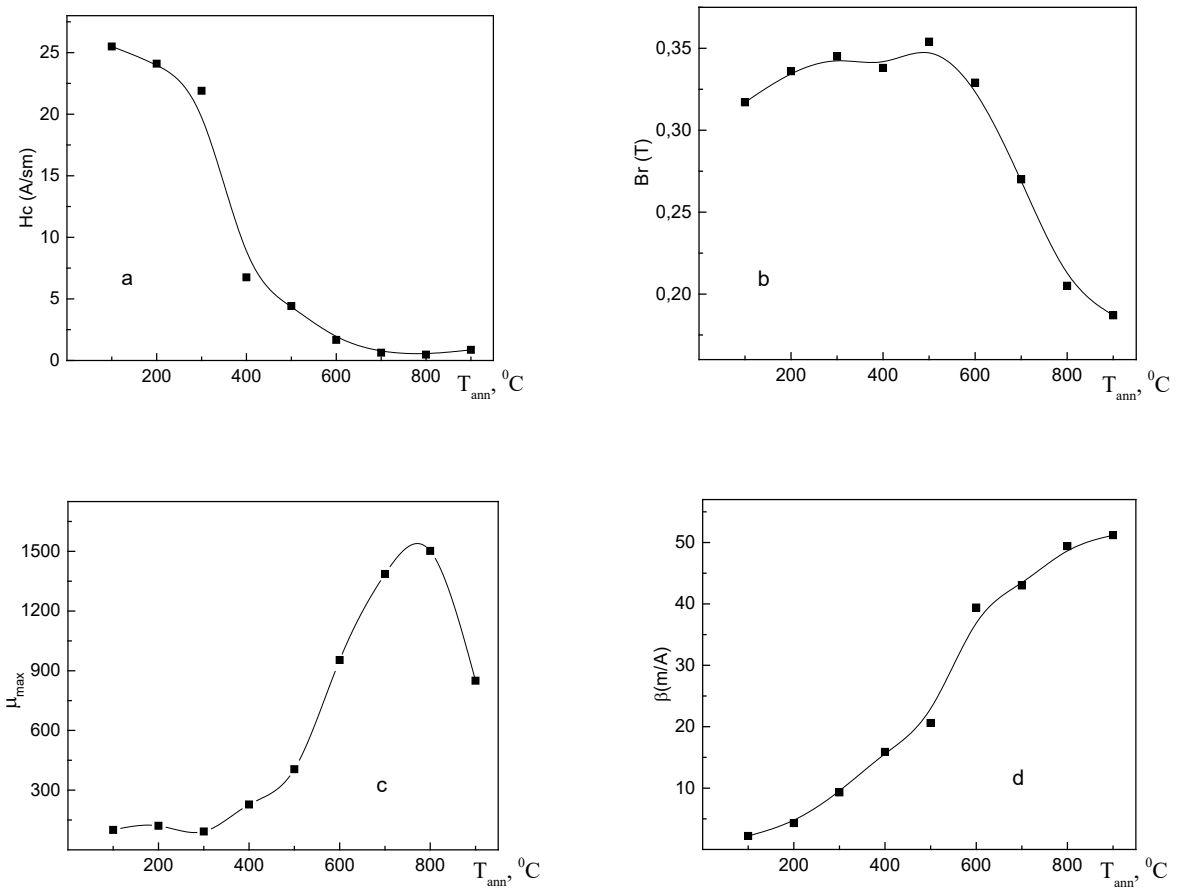

Fig. 1. Dependences of the coercive force (a), residual magnetic induction (b), maximum magnetic permeability (c), and Rayleigh coefficient (d) on the nickel annealing temperature.

Fig. 2 shows the dependence of the velocity of longitudinal ultrasonic waves on the temperature of nickel annealing. It is seen that recrystallization increases the speed of ultrasonic waves by $5.5 \%$. When annealing at more than $600{ }^{\circ} \mathrm{C}$, it was not possible to measure the wave velocity because of the high damping.

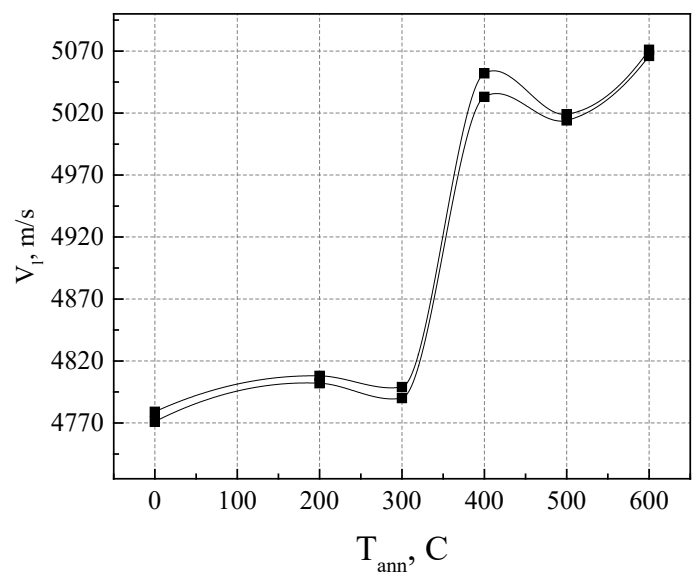

Fig. 2. The dependence of the velocity of longitudinal ultrasonic waves on the temperature of nickel annealing. 
The dependence of the ultrasonic waves damping on the $T_{\text {ann }}$ of nickel is shown in Fig. 3. An increase in the annealing temperature leads to an increase in the damping by almost 3 times. The most intense growth is observed in the range of recrystallization temperatures. This may be due to the scattering of elastic waves at the boundaries of crystal grains, when the wavelength becomes commensurate with the size of the grains grown as a result of recrystallization.

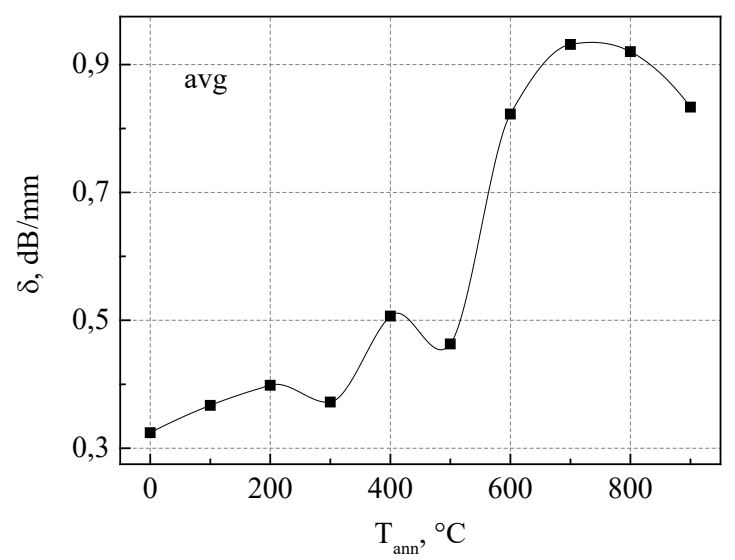

Fig. 3. Dependence of the attenuation of longitudinal ultrasonic waves on the temperature of nickel annealing.

\section{Conclusion}

Thus, it can be noted that annealing leads to very significant changes in the magnetic and acoustic properties of cold-deformed nickel. In this case, the magnetic properties are an order of magnitude more sensitive to changes in the structure and the magnitude of residual stresses in the material. Up to annealing temperatures of $700{ }^{\circ} \mathrm{C}$, the coercive force is the best diagnostic parameter. However, for a selective assessment of the structure (dislocation density, grain size) and the magnitude of residual stresses, it is advisable to determine the complex of magnetic and acoustic parameters of the material.

The research was carried out within the state assignment of Ministry of Science and Higher Education of the Russian Federation (theme "Diagnostics" No. AAAA-A18-118020690196-3)

\section{References}

1. D. O’Sullivan, M. Cotterell, S. Cassidy, D.A. Tanner, I. Mészáros, J. Magn. Magn. V. 271 (2004).

2. L. Piotrowski, B. Augustyniak, M. Chmielewski, J. Labanowski, M. Lech-Grega, NDT\&E International, V. 47 (2012).

3. V.R. Skalsky, S.I. Hirnyj, R.N. Basarab, Oil and Gas Business, No. 5, (2013).

4. V.N. Kostin, O.N. Vasilenko, D.Yu. Filatenkov, Yu. A. Chekasina, E.D. Serbin, RJNDT. V. 51 (2015).

5. M.R.N. Astudillo, M.I.L. Pumarega, N.M. Núñez, A. Pochettino, J. Ruzzante, J. Magn. Magn. V. 426 (2017). 
6. E.D. Serbin, V.N. Kostin, O.N. Vasilenko, D.G. Ksenofontov, E.G. Gerasimov, P.B. Terentev, NDT \& E International. V. 116 (2020).

7. Vonsovskiy, S.V. and Shur, Ya.S., Ferromagnetizm, (OGIZ-Gostekhizdat, Moscow, 1948) 816 p. (in Russian).

8. Tikadzumi S. Physics of ferromagnetism. Magnetic characteristics and practical applications (Mir, Moscow, 1987) 419 p. (Russian translation). 